\title{
The $(n+1) / 2$ rule for dealiasing in the split-step Fourier methods for $n$-wave interactions
}

\author{
Stanislav Derevyanko
}

\begin{abstract}
The aim of this short letter is to demonstrate that complete removal of spectral aliasing occurring due to finite numerical bandwidth used in the split-step Fourier simulations of nonlinear interactions of optical waves can be achieved by enlarging each dimension of the spectral domain by a factor $(n+1) / 2$ where $n$ is the number of interacting waves. Alternatively, when using low-pass filtering for de-aliasing this amounts to the need for filtering a $2 /(n+1)$ fraction of each spectral dimension.
\end{abstract}

Index Terms-Numerical analysis, Discrete Fourier transforms.

\section{INTRODUCTION}

$\mathbf{I}^{\mathrm{N}}$ $\mathrm{N}$ the pseudospectral (or split-step) Fourier (SSF) method of solution of nonlinear partial differential equations the solution is propagated in consecutive steps, where the linear part of the evolution operator is calculated in the Fourier space and the nonlinear step of the evolution is performed separately in the coordinate space [1], [2]. The transition between the Fourier and coordinate space is performed by the Fast Fourier Transform which requires only $O\left(N \log _{2} N\right)$ operations. This makes SSF the ever-attractive tool for numerical modelling of single and multidimensional nonlinear systems arising in different fields of photonics [2]. The important and well known drawback of all spectral methods (including SSF) is aliasing which comes from the inability of the scheme to resolve the wave vectors (or frequencies if the problem is posed in the time domain) beyond the computational boundaries. Indeed if $N$ discrete wave vectors are chosen within the interval $\left[-K_{\max }, K_{\max }\right]$ then the modes with the wave vectors outside the computational region will be automatically mapped (aliased) back to the computational domain modulo $2 K_{\max }$, that is to say that a mode with the wave vector $k^{\prime},\left|k^{\prime}\right|>K_{\max }$ is undistinguishable from a mode $k^{\prime}+2 m K_{\max }$ from inside the domain, where $m$ is an integer and $m \neq 0$. Such modes appear due to spectral broadening when one computes the nonlinear step of the evolution in the SSF scheme. If the system is characterised by nonlinear processes of $n$-th order (e.g. via the nonlinear electric susceptibility $\chi^{(n)}$ ) then the $n$ wave interaction of the modes leads to the computationally resolved wave vectors $k$ inside the interval $\left[-K_{\max }, K_{\max }\right]$ being mapped into the wider region $\left[-n K_{\max }, n K_{\max }\right]$ and all the modes occupying the shell $K_{\max }<|k|<n K_{\max }$ are spuriously mapped back into the computational domain which constitutes the effect of aliasing. When the level of nonlinearity is low and the bandwidth of the signal is easily controlled during the simulation the aliasing does not present a

S. Derevyanko is with the Photonics Research Group, Aston University, Aston Triangle, Birmingham B4 7ET, United Kingdom significant problem if one keeps the computational bandwidth high enough so that the aliased modes have low power and do not distort the solution (spatial and temporal solitons and beams provide a good example of such class of systems). However when working with broadband signals (e.g. systems characterised by broadband noise, wavelength-divisionmultiplexing (WDM) systems, supercontinuum generation etc) aliasing can present a problem and can potentially lead to false numerical instabilities.

The problem of aliasing in the pseudospectral modelling of nonlinear systems first occurred in computational fluid dynamics with regard to the direct numerical simulations (DNS) of the Navier-Stokes equation and problems of climate modelling. Various remedies have been suggested since [1], [3], [4], [5] and here I will only consider "padding" technique where prior the computation of the nonlinear step the Fourier domain is artificially enlarged to accommodate all modes resulting from the $n$-wave interaction which would otherwise be spuriously aliased. Before and after computing the nonlinear step the unresolved extra modes with $|k|>K_{\max }$ are set to zero (i.e. filtered). Since the enlargement of the computational domain incurs a computational time overhead the question then arises of what is the minimal number of extra modes one has to introduce to dispense from the aliasing completely. From the reasoning above one can see that the $n$ fold enlargement of the Fourier domain from $\left[-K_{\max }, K_{\max }\right]$ to $\left[-n K_{\max }, n K_{\max }\right]$ is sufficient to filter out all the spurious modes. However it is clear that such broad filtering is wasteful. For the Navier-Stokes equation (which has a second order nonlinearity, $n=2$ ) Orszag [7] showed that it is sufficient to enlarge the computational domain by a factor of $3 / 2$ instead of 2 because the remaining quarter of the modes will be aliased back into the filter region and hence will not survive anyway. Note that the method of "padding" is equivalent to low pass spectral filtering where instead of enlargement of the spectral domain the aliased high-wavenumber modes are filtered out at each linear SSF step. In this case Orszag's rule suggests that it is sufficient to filter just one third of each spectral domain and not half of it as one would expect from a naive reasoning.

In the nonlinear photonics one usually deals with $\operatorname{Kerr}(n=$ 3 ) and sometimes higher nonlinearities. Therefore a generic padding (or filtering) rule is required for higher number of interacting waves, i.e. for higher order nonlinearities. The next section provides such a criterion.

$$
\text { II. THE “ }(n+1) / 2 \text { ” RULE }
$$

In this section I propose a simple generalisation of the Orszag's " $3 / 2$ rule" to systems with arbitrary order of non- 
linearity, $n$. The problem can be reformulated as follows: given the computational Fourier domain $\left[-K_{\max }, K_{\max }\right]$, we introduce the de-aliasing passband filter that only keeps the modes inside the interval $\left[-\alpha K_{\max }, \alpha K_{\max }\right],(0<\alpha<1)$. The question is then what is the maximum achievable relative bandwidth of such a filter, $\alpha$, such that all unfiltered modes still remain de-aliased. Once such $\alpha_{\max }$ is found one can then enlarge the computational domain prior to computing the nonlinear step by the factor of $1 / \alpha_{\max }$ so that the Fourier resolved (and physically meaningful) modes are always dealiased. Alternatively one can keep the domain unchanged but filter out the top $1-\alpha_{\max }$ fraction of the spectrum at a price of the corresponding loss of resolution. The naive wasteful filtering gives an estimate $\alpha_{\max }=1 / n$ which means that in the case of $n=3$ two thirds of each computational dimension is wasted and one has to use trice as many modes to maintain the same resolution. Clearly one needs a more optimal estimate - an analogue of Orszag's $\alpha_{\max }=2 / 3$ for $n=2$.

Consider the domain of unfiltered modes $\left[-\alpha K_{\max }, \alpha K_{\max }\right]$. Due to $n$-wave interaction these modes are mapped into a wider region $\left[-\alpha n K_{\max }, \alpha n K_{\max }\right]$ and all the resulting modes inside the shell $K_{\max }<|k|<\alpha n K_{\max }$ will be mapped back into $\left[-K_{\max }, K_{\max }\right]$ potentially aliasing modes inside the unfiltered interval $\left[-\alpha K_{\max }, \alpha K_{\max }\right]$. Let us denote such an aliasing mode as $k^{\prime}=\beta K_{\max }$ where $1<|\beta|<\alpha n$. Then the condition that the mode $k^{\prime}$ resulted from the nonlinear interaction of $n$ modes within the unfiltered region is mapped back into the unfiltered region is equivalent to the existence of a non-zero integer $m$, such that the following inequalities are met:

$$
-\alpha K_{\max }<k^{\prime}+2 m K_{\max }<\alpha K_{\max }, \quad|m|=1,2, \ldots
$$

or equivalently:

$$
-\alpha-\beta<2 m<\alpha-\beta, \quad|m|=1,2, \ldots
$$

Finding the optimal filter bandwidth amounts now to finding such maximum value of $\alpha$ for which condition (2) is never met, i.e. no $n$ modes within the unfiltered region are able to produce a mode that is aliased back into the same region. One can readily see that no non-zero $m$ satisfying inequalities (2) exists if the r.h.s is less than 2 and the l.h.s. is greater than -2 . Recalling that $|\beta|<\alpha n$ we obtain a sufficient criterion for full de-aliasing:

$$
\alpha<\frac{2}{n+1},
$$

which yields $\alpha_{\max }=2 /(n+1)$. The Orszag criterion when $n=2$ follows.

\section{NUMERICAL EXAMPLE}

Let us illustrate the practical concept of full de-aliasing with a simple numerical example. The effect of spurious aliasing is especially pertinent in the situation where significant spectral broadening occurs while the spectral intensity remains more or less fixed. As an example of such a situation I take the propagation of a chirped pulse in a single mode optical fiber in the regime where self-phase-modulation (SPM) dominates.

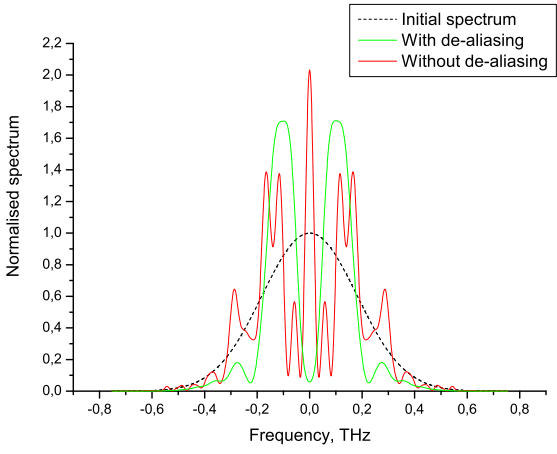

Fig. 1. (Color online) Spectral evolution of a chirped Gaussian pulse with (green line) and without (red line) de-aliasing. The initial spectrum is given as dashed line.

The pulse evolution is given by the 1D Nonlinear Schrodinger equation (NSE) [2]:

$$
\mathrm{i} \frac{\partial u}{\partial z}-\frac{\beta_{2}}{2} \frac{\partial^{2} u}{\partial t^{2}}+\gamma|u|^{2} u=0
$$

where $u(z, t)$ is the electromagnetic field and $\beta_{2}$ and $\gamma$ are the dispersion and nonlinearity coefficients respectively. As for the initial condition I opt for a chirped Gaussian pulse characterized by the peak power, $P_{0}$, width, $T_{0}$, and the dimensionless chirp parameter, $C$ :

$$
u(0, t)=\sqrt{P_{0}} \exp \left[-\frac{1+\mathrm{i} C}{2} \frac{t^{2}}{T_{0}^{2}}\right]
$$

The two important scales of propagation are the nonlinear length $L_{N L}=\left(\gamma P_{0}\right)^{-1}$ and the dispersion length $L_{D}=$ $T_{0}^{2} /\left|\beta_{2}\right|$. On the scales of the order of $L_{N L}$ the SPM effects result in the spectral broadening of the pulse (which is assisted by the presence of chirp) while the anomalous linear dispersion (acting on the scales $\sim L_{D}$ ) prevents the spectral power from dispersing. In our simulation the following parameters were used: $\beta_{2}=-5 \mathrm{ps}^{2} / \mathrm{km}, \gamma=3 \mathrm{~W}^{-1} \mathrm{~km}^{-1}, T_{0}=20 \mathrm{ps}$, $P_{0}=40 \mathrm{~mW}, C=5$. This yields $L_{N L}=8.3 \mathrm{~km}$ and $L_{D}=80 \mathrm{~km}$. The pulse is propagated over the distance of one dispersion length $L=80 \mathrm{~km}$. The initial full width at half minimum (FWHM) bandwidth of the pulse is $B=$ $2(\ln 2)^{1 / 2} / T_{0} \approx 83 \mathrm{GHz}$. In the simulation I used $M=1024$ Fourier modes with the mode spacing of $1.47 \mathrm{GHz}$ so that the initial computational bandwidth is $B_{c}=1.5 \mathrm{THz}$. Two runs were performed: with and without de-aliasing (see Fig. 1). The de-aliasing is achieved by increasing the number of modes $M$ by a factor of 2 (as prescribed by a $(n+1) / 2$ rule for $n=3$ ) at each nonlinear step. One can observe that even in the case when the computational bandwidth $B_{c}$ encompasses the broadened spectrum, the effects of spurious aliasing result in the inadequate description of the evolving pulse (red line in Fig.1). These effects are efficiently removed by spectral padding as shown by the green line in the same figure.

One can argue, of course, that the effects of aliasing can also be removed by choosing large enough number of modes, $M$ (and hence large enough computational bandwidth $B_{c}$ ) while keeping the mode spacing fixed, so that the aliased modes will 
be of no significance at all (since they will be produced from the interaction of negligibly small modes from the wings of the spectrum). While this is true it is also clear that such an "overkill" solution is wasteful since larger arrays take longer to process. Moreover, there is no a-priory guideline as to how large the number $M$ should be to suppress the aliasing. On the other hand the $(n+1) / 2$ rule provides a clear recipe as to how one should enlarge the number of modes (given a fixed spectral resolution $M$ ) at each nonlinear step without the need for blind guessing or using redundantly large arrays.

\section{CONCLUSION}

The obtained simple result can be summarised in the following statement: In order to achieve full de-aliasing in the SSF scheme with the computational domain $\left[-K_{\max }, K_{\max }\right]$ in a system with $n$-th order nonlinearity the domain must be enlarged by a factor of $(n+1) / 2$ prior to the nonlinear step and the aliased modes within the shell $K_{\max }<|k|<$ $K_{\max }(n+1) / 2$ must be filtered (padded with zeros) prior to the nonlinear step and discarded afterwards. An alternative statement is that if one employs low pass filtering without enlarging the computational domain then it is sufficient to keep a fraction $\alpha_{\max }=2 /(n+1)$ of the modes to ensure full dealiasing - the rest of the modes remain unresolved. One can see that the higher the nonlinearity the higher the cost, which is not surprising in view the higher harmonics generation. For systems with Kerr nonlinearity each spectral dimension must be enlarged by a factor of 2 (or alternatively half of each domain must be filtered out) to achieve full de-aliasing. I have also illustrated the benefits of the full de-aliasing method by providing a simple numerical example of pulse propagation in an optical fiber.

Finally the dimensionality of the problem does not enter the argument above, or more precisely, for multidimensional nonlinear systems this criterion applies to each dimension separately, so for a $d$-dimensional system the computational FFT overhead imposed by the enlargement of the array size scales as $((n+1) / 2)^{d}$. If one chooses to apply spectral filtering this amounts to effective domain reduction by the same factor. For the 1D problem of pulse propagation in an optical fiber half of the spectral data must be discarded to prevent aliasing. The situation tends to get worse as one considers higher dimensional problems (e.g. optical beams and optical bullets). Still, compared to the wasteful filtering with $\alpha_{\max }=1 / n$ one gets an improvement in resolution by a factor of $(2 n /(n+1))^{d}=(3 / 2)^{d}$ for systems with Kerr nonlinearity.

\section{REFERENCES}

[1] J. P. Boyd, Chebyshev and Fourier spectral methods, 2nd ed. Mineola, NY: Dover, 2000.

[2] G. P. Agrawal, Nonlinear Fiber Optics, 4th ed. San Diego, CA: Academic Press, 2007.

[3] G.S. Patterson and S.A. Orszag, "Spectral calculations of isotropic turbulence: effecto of removal of aliasing interactions", Phys. Fluids, vol. 14 , pp. $2538-2541,1971$

[4] S. Lele, "Compact finite diference schemes with spectral like resolution", J. Comp. Phys., vol. 103, pp. 16-42, 1992.

[5] G.A. Blaisdell, E.T. Spyropoulos and J.H. Qin, "The effect of the formulation of nonlinear terms on aliasing errors in spectral methods", Appl. Numer. Math., vol. 21, pp. 207 - 219, 1996.
[6] N.A. Phillips, "An example of nonlinear compuatational instability," in The Atmosphere and Sea Motion, B. Bolin ed., New York: Rockefeller Inst. Press, 1959.

[7] S.A. Orszag, "On the elimination of aliasing in finite-difference schemes by filtering high-wavenumber components", J. Atmos. Sci., vol. 28, pp. 1074-1074, 1971.

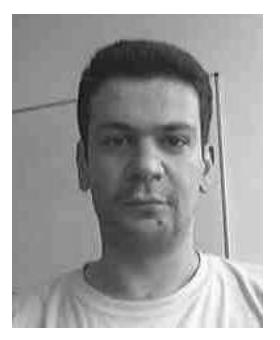

Stanislav Derevyanko received his M. Sc. from Kharkov State University in 1999 and later obtained a Ph.D. in theoretical physics from Institute for Radiophysics and Electronics, Kharkov, Ukraine, in 2001. In 2002 he joined Photonics Research Group at Aston University, Birmingham, UK. His research interests include the effects of noise on optical communication systems, Monte-Carlo simulations, integrable systems, nonlinear and stochastic dynamics. 